\title{
Metals in liver and kidney tissues of autumn- migrating dunlin Calidris alpina and curlew sandpiper Calidris ferruginea staging at the Baltic Sea
}

\author{
Sven Blomqvist ${ }^{1}$, Adrian Frank ${ }^{2} \&$ Lars R. Petersson ${ }^{2}$ \\ ${ }^{1}$ Askö Laboratory and Department of Geology, University of Stockholm, S-106 91 Stockholm, Sweden \\ ${ }^{2}$ Department of Chemistry, National Veterinary Institute, S-750 07 Uppsala, Sweden
}

\begin{abstract}
Concentrations of 10 metals ( $\mathrm{Ca}, \mathrm{Cd}, \mathrm{Co}, \mathrm{Cu}, \mathrm{Fe}, \mathrm{Mg}, \mathrm{Mn}, \mathrm{Pb}, \mathrm{V}$ and $\mathrm{Zn}$ ) were determined in liver and kidney tissues of dunlin Calidris alpina $(n=70)$ and curlew sandpiper Calidris ferruginea $(\mathrm{n}=28)$. Element associations are reported. Significant linear correlations were found between renal and hepatic concentrations of cadmium, copper, magnesium, and manganese. Copper showed an agerelated concentration decrease, whereas cadmium concentration increased with age. Mean biological half-life of renal cadmium in dunlin is estimated at about $1 \mathrm{yr}$ (with an implied maximum of $2.5 \mathrm{yr}$ ). The potential of cadmium as a future environmental hazard to aquatic birds is discussed
\end{abstract}

\section{INTRODUCTION}

Two characteristic biological features of estuarine and muddy intertidal areas are high primary production (Woodwell et al. 1973) and high zoobenthic production (Wolff 1983). A considerable proportion of the intertidal zoobenthic production may be consumed by shorebirds (Baird et al. 1985). Levels of certain heavy metals in the sediment and its invertebrate fauna in these coastal environments are often elevated, naturally or through anthropogenic contamination (Förstner \& Müller 1974, Förstner 1980, Förstner \& Whittmann 1981, Bryan 1984, Moore \& Ramamoorthy 1984, Salomons \& Förstner 1984). Thus, these are habitats where bioaccumulation and food chain transfer of metals can be expected to be of significance, with metal accumulation in shore-living birds possibly reaching levels high enough to affect individuals or populations adversely.

Fairly little has been published previously on contaminant exposure and homeostatic concentration of trace metals in coastal birds (Eisler 1981). Waders (Charadrii) are prominent and numerous birds subsisting on the invertebrate fauna of intertidal coastal sediments. Some species are resident throughout the year, but most migrate north for breeding. Waders may transfer metals from the aquatic to the terrestrial environment, and may also transport contaminants to remote areas. Small and intermediate wader species are frequently eaten by certain birds of prey during their breeding season (Cade 1960, Sulkava 1968, White \& Cade 1971, Hautala \& Sulkava 1977, Lindberg 1983), migration (Dekker 1980, Andersson 1985a, b) and in the winter quarters (Page \& Whitacre 1975, Clunie 1976, Mearns 1982, Kus et al. 1984, Whitfield 1985), and may thus be sources of metal exposure to such raptors.

Among the smaller waders, the genus Calidris is abundant in both the Old and the New World, breeding mostly in the Taiga, sub-Arctic and Arctic regions (Dement'ev \& Gladkov 1969, Glutz von Blutzheim et al. 1975, Johnsgard 1981). In the non-breeding season, which comprises about 10 mo of the year, they are usually found in muddy and sandy estuarine and coastal environments, but they also occur on rocky shores with seaweed accumulations (Summers et al. 1977), and inland along shallow rivers and lakes. Only 2 Calidris species, the purple sandpiper C. maritima of the North Atlantic and the rock sandpiper C. ptilocnemis of the North Pacific, are regularly found on bare rocky seashores during the non-breeding season (Gabrielson \& Lincoln 1959, Cramp \& Simmons 1983). 
Although the number of publications on the genus Calidris has increased considerably during recent decades (Blomqvist 1985), documentation of trace element levels in these birds is still scanty. Earlier studies in England and Denmark considered only one (Parslow 1973, Clausen et al. 1985) or a few heavy metals (Ward 1979, Evans \& Moon 1981, NERC 1983). In a study from Texas, United States, heavy metals and selenium were determined either in livers or in kidneys of unaged birds in one season (White et al. 1980). Concentrations and linear correlations of some elements in certain tissues and glands have previously been reported from birds collected in the Netherlands and Sweden (Goede 1985, Goede \& de Bruin 1985a, b, Goede \& de Voogt 1985). However, these studies were mainly focused on feathers as indicators of trace element exposure (see also Goede \& de Bruin 1984). In view of this paucity of data, we have determined concentrations of 10 metals in the liver and kidneys of 2 Calidris species collected at a staging area in the Baltic Sea.
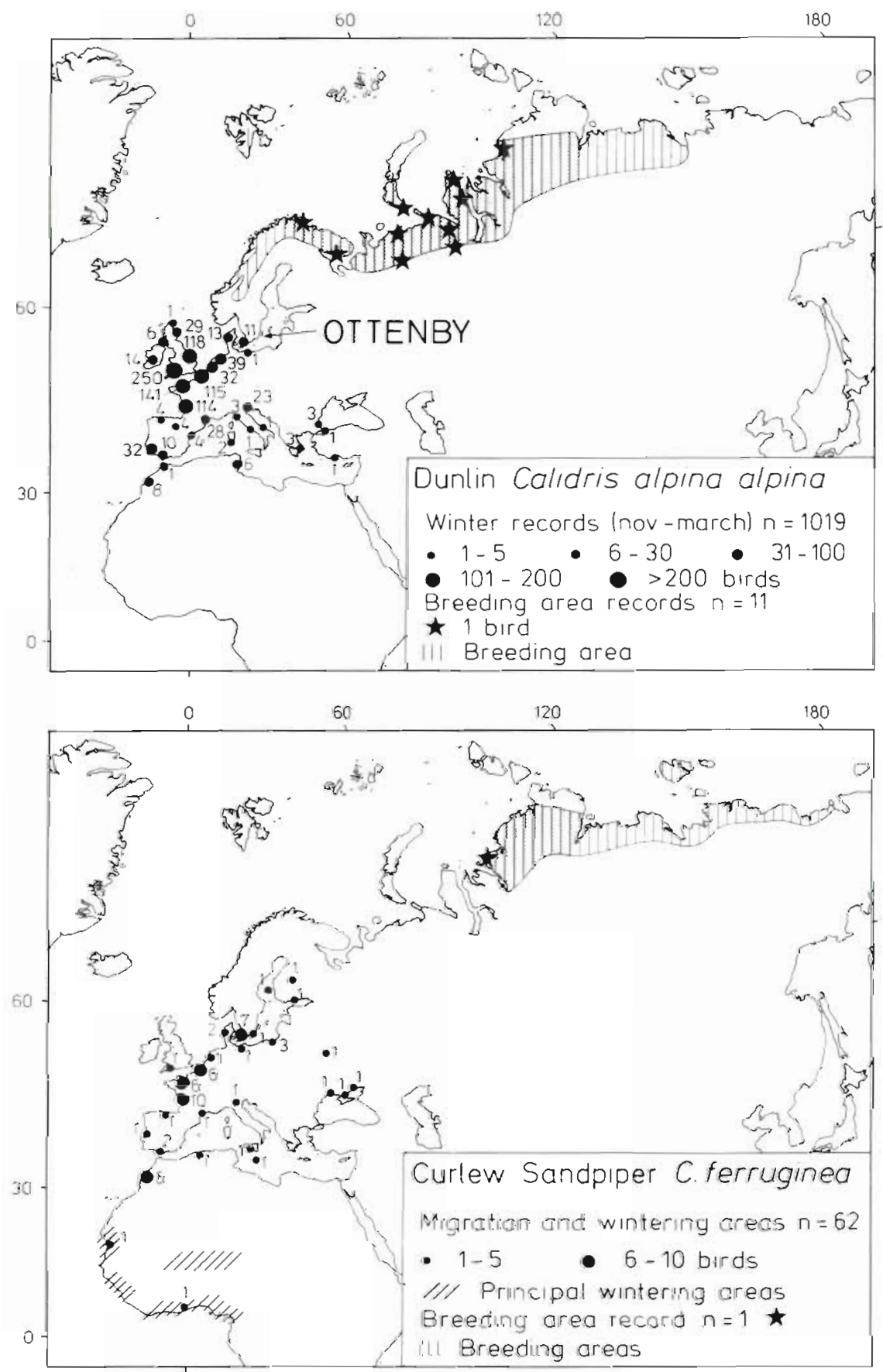

Fig. 1. Calidris alpina alpina with $C$. a. centralis, and $C$. ferruginea. Breeding range (vertical hatching) of dunlin and curlew sandpiper (sources: SOF 1978, Hildén \& Hyytiä 1981, Kâlás \& Byrkjedal 1981, Flint et al. 1984, Greenwood 1986). Shaded areas: regions from which birds passing Ottenby probably originate; stars: recoveries/controls in breeding areas; dots: records in migration and wintering areas, of birds ringed at Ottenby. Numbers: number of records in the specific areas (sources: Liljefors et al. 1985, Pettersson et al. 1986, J. Pettersson pers. comm.). Principal wintering areas of curlew sandpipers passing Ottenby are indicated by crossed hatching (sources: Wilson et al. 1980, Fournier \& Dick 1981). Map projection: van der Grinten 


\section{MATERIAL AND METHODS}

During the autumns of 1981 to 1983 we collected specimens of dunlin Calidris alpina alpina L. and curlew sandpiper Calidris ferruginea Pont. at Ottenby Bird Observatory $\left(56^{\circ} 12^{\prime} \mathrm{N}, 16^{\circ} 24^{\prime} \mathrm{E}\right)$, situated on the southernmost point of the Swedish island of Oland, in the Baltic Sea (Fig. 1). In autumn, large numbers of migrant birds, mainly from the northeast (i.e. Finland and the USSR), cross this area (Svärdson 1953, Edelstam 1972, Prater 1980, Liljefors et al. 1985).

The species. Except for winter area selection and first-year summering habits, the 2 species are rather similar. Their different age groups tend to pass Ottenby at the same time in the autumn (Edelstam 1972). Their breeding areas in the far north of Siberia partially overlap. The subspecies of dunlin named above breeds from northern Fenno-Scandia to well east of the Taimyr peninsula (Fig. 1). The curlew sandpiper's entirely Siberian breeding range reaches from the Yenisey to slightly east of the Kolyma (Fig. 1). Both species breed primarily in tussock and hummocky peat-tundra (Haviland 1915, Portenko 1959, Uspenski 1969).

The 2 species deviate with respect to wintering areas and summer habitats of first-year birds. This dunlin subspecies winters in the temperate climate zone, mainly in coastal areas in western Europe, particularly in the British Isles (Fig. 1; Hardy \& Minton 1980, Greenwood 1984, Symonds \& Langslow 1984). Some dunlins already return to northern areas in their first year, but the majority probably do not start breeding until they are 2 yr old (i.e. in their third calendar year) (Soikkeli 1967, 1970). Curlew sandpipers passing Ottenby follow the East Atlantic Flyway (sensu Altenburg et al. 1982, p. 93) with tropical West Africa and the Gulf of Guinea as destinations (Fig. 1; Wilson et al. 1980 , Fournier \& Dick 1981). It has been suggested that the spring migration of the West African curlew sandpipers traces a more easterly route (Nørrevang 1959, Stanley \& Minton 1972, Elliott et al. 1976, Wilson et al. 1980), i.e. a route following a great circle back to their breeding grounds (Grimes 1974). Large concentrations of curlew sandpiper also occur in coastal areas of Namibia and South Africa (Summers et al. 1977, Whitelaw et al. 1978, Ryan \& Cooper 1985). These birds probably do not follow the East Atlantic Flyway, but take a more continental eastern route (Elliott et al. 1976, Wilson et al. 1980). Curlew sandpipers spend their first year in the south (Elliott et al. 1976), and in this respect they differ from dunlins, where at least some first-year birds go north in summer.

Collection. The autumn-migrating birds in this study were caught in cage-traps (Bub 1971, p. 201), when staging on the banks of decaying, stranded sea- weed fringing the shores at Ottenby. According to the colour of plumage, the degree of feather wear, and the moult stage, they were aged into 3 classes: juveniles (0.1 yr old), first-year birds (second calendar year birds, i.e. $1.1 \mathrm{yr}$ ) and adults (older than first-year birds) (terminology following Mead 1985, p. 6). Age criteria used were those given by Prater et al. (1977). The mean lifespan of a fledged dunlin has been estimated as $5.3 \mathrm{yr}$ (Soikkeli 1970). However, dunlins, as well as curlew sandpipers, may reach a considerably greater age. The maximum age recorded for a dunlin is $23.7 \mathrm{yr}$ (Staav 1983) and for a curlew sandpiper 12.2 yr (Dejonghe \& Czajkowski 1983).

Sample handling. Captured birds were killed by asphyxiation, temporarily stored in a refrigerator, and air-freighted in a cooling box. Tissue samples were obained by necropsy at the National Veterinary Institute in Uppsala within $48 \mathrm{~h}$ of capture. Necropsy, sample preparation and chemical analysis were performed on 70 dunlins and 28 curlew sandpipers.

Liver and kidneys were dissected and immediately weighed. The organ samples were placed in small plastic boxes and stored at $-20^{\circ} \mathrm{C}$ until analysed. Liver tissue samples of 2 to $4 \mathrm{~g}$ and kidney tissue samples of 0.5 to $1 \mathrm{~g}$ were wrapped in a filter paper and pretreated by automatic wet digestion (Frank 1976). A mixture of $65 \%$ nitric acid and $70 \%$ perchloric acid ( $7: 3$ by vol.) was used as an oxidizing agent. The digestion was performed in borosilicate glass tubes overnight, using an electrically heated aluminium block connected to a microprocessor for control of temperature and time (Tecator Digestion System, Model 40; Tecator AB, Höganäs, Sweden). The residue was evaporated to dryness and dissolved in $10 \mathrm{ml}$ (or in the case of limited sample amount as for the kidneys, in $3 \mathrm{ml}$ ), ionic buffer (Frank \& Petersson 1983). Chemical analysis was performed by simultaneous multielement analysis of 10 elements $\left(\mathrm{Ca}, \mathrm{Cd}, \mathrm{Co}, \mathrm{Cu}_{1} \mathrm{Fe}\right.$, $\mathrm{Mg}, \mathrm{Mn}, \mathrm{Pb}, \mathrm{V}$ and $\mathrm{Zn}$ ), using a direct current plasmaatomic emission spectrometer, Model SpectraSpan III A (Beckman Instruments Inc., Irvine, California, USA), as described by Frank \& Petersson (1983). Detection limits in the liver and kidney tissues are reported in Table 1. Tissues concentrations are given as $\mu \mathrm{g}$ per $\mathrm{g}$ wet tissue throughout.

Data handling. Since data on environmental contaminant concentrations are often non-normally distributed (Liebscher \& Smith 1968, Esmen \& Hammad 1977, Talbot \& Simpson 1983), we have, in addition to the arithmetic means and standard deviations, calculated the geometric means and the medians of the data sets. Parametric tests, and rank and linear conrelations were performed by SPSS computer programmes (Nie et al. 1975), and the non-linear regressions by SAS software (Allen Rey 1982). Descriptive statistics and 
Table 1. Detection limit ( $\mathrm{DL}, \mu \mathrm{g} \mathrm{g} \mathrm{g}^{-1}$ wet weight) for the 10 elements determined in $2 \mathrm{~g}$ of liver and $0.5 \mathrm{~g}$ of kidney tissue. In most analyses performed the DL's are lower than in this table, since the digested sample commonly exceeded these minimum amounts of tissue

\begin{tabular}{|lll|}
\hline Metal & Liver & Kidney \\
\hline $\mathrm{Ca}$ & 1.34 & 1.61 \\
$\mathrm{Cd}$ & 0.06 & 0.07 \\
$\mathrm{Co}$ & 0.020 & 0.024 \\
$\mathrm{Cu}$ & 0.03 & 0.04 \\
$\mathrm{Fe}$ & 0.15 & 0.18 \\
$\mathrm{Mg}$ & 0.10 & 0.11 \\
$\mathrm{Mn}$ & 0.04 & 0.05 \\
$\mathrm{~Pb}$ & 0.06 & 0.07 \\
$\mathrm{~V}$ & 0.015 & 0.018 \\
$\mathrm{Zn}$ & 0.73 & 0.88 \\
\hline
\end{tabular}

associated tests employed can be found in Sokal \& Rohlf (1981). Ages of all birds are converted to decimals. Avian nomenclature follows Voous (1973).

\section{RESULTS AND DISCUSSION}

\section{General}

Concentrations of 9 metals determined in the liver and kidney tissues of the different age classes of dunlins and curlew sandpipers are summarized in Table 2. Vanadium has been excluded from this table since its concentration levels were often below or close to the detection limit (highest records in liver and kidney tissue are 0.04 and $0.06 \mathrm{ppm}$, respectively). The data in the table have not been separated into groups between years, since the differences are only slight. As shown in Table 2, the metal levels are generally low. In both species, the arithmetic means of 4 elements - copper, iron, manganese and zinc - are significantly higher in liver than in kidney tissue, whereas the opposite is true for cadmium (statistical criteria: 2-tailed $t$-test, $\mathrm{p}<0.005)$.

\section{Correlation}

Inter-element correlations within liver and kidney tissues, respectively, and element correlations between these tissues were calculated by Kendall rank-order correlation coefficient $(\tau)$ (Tables 3 \& 4). Cobalt and lead have been excluded from these correlation coefficient matrixes since their recorded concentrations were low and their ranges small (Table 2). In both species, statistically significant inter-tissue correlations within elements were found for 4 metals cadminm, copper, magnesium and manganese - and these were linearly related (Fig. 2). The correlations noted for cadmium and copper were also linked to agerelated concentration changes (see below); apart from. these 2 elements, no statistically significant agerelated changes of metal levels were detected. Most inter-element correlations found in both species (Tables $3 \& 4$ ) have prcviously been reported from other organisms, and have also been proposed by various biochemical or physiological interacting mechanisms. This applies to cadmium-calcium and cadmium-iron (Chang et al. 1981, Neathery 1981) and copper-iron, copper-manganese and copper-zinc (Kirchgessner et al. 1979). In birds, a positive linear correlation between copper and zinc has been noted in liver tissue of the brown pelican Pelecanus occidentalis (Ohlendorf et al. 1985).

\section{Copper}

For copper, the arithmetic and geometric mean and the median hepatic and renal levels were significantly higher $(19$ to $30 \%)$ in juveniles than in older individuals. This trend of age-related decrease (Fig. 3) is statistically highly significant for kidney tissues of both species and for liver tissue of dunlin (2-tailed $t$-tests, $p<0.001$ ), but is less apparent in the liver of curlew sandpiper $(p<0.07)$. A decreasing trend of copper with age is consistent with some findings on mammals (Widdowson \& Dickerson 1964, Martin et al. 1976, Duinker et al. 1979), but, apart from one report on liver tissue in the osprey Pandion haliaetus (Wiemeyer et al. 1980), such trends have not been reported in wild birds before. As mentioned above, the renal versus hepatic copper concentrations were linearly correlated in both species (Fig. 2), with a regression coefficient of about 0.4 . This deviates from a study on the mute swan Cygnus olor where the slope $(0.0024)$ borders on insignificance (Frank \& Borg 1979). A positive linear correlation between hepatic and renal copper concentrations has recently also been reported in the brown pelican (Ohlendorf et al. 1985).

\section{Cadmium}

The cadmium concentration estimates derived as arithmetic or geometric means or medians have a fairly similar pattern in both species (Table 2), with a pronounced age-related organ-specific increase (Fig.3). This is highest for the kidney tissues, with an average 17.6 and 28.9-fold increase $(p<0.001)$ (calculated on the arithmetic means and tested by 2 -tailed $t$-test) between juveniles and adults of dunlin and curlew sandpiper, respectively. Corresponding factors for the liver tissues are $5.3(\mathrm{p}<0.001)$ and $12.6(\mathrm{p}<0.005)$. 
Table 2. Calidris alpina and C. ferruginea. Minor and trace element concentrations ( $\mu \mathrm{g} \mathrm{g}^{-1}$ fresh wt) in liver and kidney tissue of dunlin and curlew sandpiper Median ( $\left.P_{50}\right)$, geometric mean (GM), arithmetic mean (AM) and one standard deviation (SD), as well as range are given. Dashes in the GM column indicate non-calculable means due to zero values

\begin{tabular}{|c|c|c|c|c|c|c|c|c|c|c|c|c|c|}
\hline \multirow[t]{2}{*}{ Element } & \multirow[t]{2}{*}{ Age } & \multicolumn{6}{|c|}{ Liver } & \multicolumn{5}{|c|}{ Kidney } & \multirow[b]{2}{*}{ Range } \\
\hline & & $n$ & $P_{500}$ & GM & $\mathrm{AM}$ & $\mathrm{SD}$ & Range & $\mathrm{n}$ & $P_{s u}$ & GM & $A M$ & $\mathrm{SD}$ & \\
\hline \multicolumn{14}{|l|}{ Dunlin } \\
\hline $\mathrm{Ca}$ & $\begin{array}{l}\text { Overall } \\
\text { Juvenile } \\
\text { First-year } \\
\text { Adult }\end{array}$ & $\begin{array}{l}69 \\
17 \\
27 \\
25\end{array}$ & $\begin{array}{l}47 \\
53 \\
45 \\
43\end{array}$ & $\begin{array}{l}50 \\
56 \\
53 \\
43\end{array}$ & $\begin{array}{r}74 \\
57 \\
113 \\
44\end{array}$ & $\begin{array}{r}211 \\
12 \\
337 \\
11\end{array}$ & $\begin{array}{l}24-1800 \\
39-78 \\
28-1800 \\
24-70\end{array}$ & $\begin{array}{l}69 \\
17 \\
25 \\
27\end{array}$ & $\begin{array}{l}56 \\
68 \\
49 \\
57\end{array}$ & $\begin{array}{l}75 \\
95 \\
67 \\
71\end{array}$ & $\begin{array}{l}202 \\
273 \\
212 \\
150\end{array}$ & $\begin{array}{l}510 \\
627 \\
585 \\
339\end{array}$ & $\begin{array}{l}32-2700 \\
48-2460 \\
36-2700 \\
32-1550\end{array}$ \\
\hline $\mathrm{Cd}$ & $\begin{array}{l}\text { Overall } \\
\text { Juvenile } \\
\text { First-year } \\
\text { Adult }\end{array}$ & $\begin{array}{l}70 \\
17 \\
27 \\
26\end{array}$ & $\begin{array}{l}0.21 \\
0.08 \\
0.26 \\
0.28\end{array}$ & $\begin{array}{l}- \\
\overline{-} \\
0.29 \\
0.28\end{array}$ & $\begin{array}{l}0.26 \\
0.06 \\
0.33 \\
0.32\end{array}$ & $\begin{array}{l}0.20 \\
0.06 \\
0.18 \\
0.18\end{array}$ & $\begin{array}{r}<0.06-1.01 \\
<0.06-0.13 \\
0.12-0.83 \\
0.13-1.01\end{array}$ & $\begin{array}{l}69 \\
17 \\
25 \\
27\end{array}$ & $\begin{array}{l}0.66 \\
0.00 \\
0.71 \\
0.95\end{array}$ & $\begin{array}{c}- \\
\overline{-} \\
0.59 \\
0.96\end{array}$ & $\begin{array}{l}0.76 \\
0.07 \\
0.72 \\
1.23\end{array}$ & $\begin{array}{l}0.77 \\
0.10 \\
0.43 \\
0.92\end{array}$ & $\begin{array}{r}<0.07-4.08 \\
<0.07-0.30 \\
0.15-1.66 \\
0.14-4.08\end{array}$ \\
\hline $\mathrm{Co}$ & $\begin{array}{l}\text { Overall } \\
\text { Juvenile } \\
\text { First-year } \\
\text { Adult }\end{array}$ & $\begin{array}{l}70 \\
17 \\
27 \\
26\end{array}$ & $\begin{array}{l}0.04 \\
0.04 \\
0.04 \\
0.04\end{array}$ & $\begin{array}{l}- \\
- \\
- \\
-\end{array}$ & $\begin{array}{l}0.04 \\
0.03 \\
0.03 \\
0.04\end{array}$ & $\begin{array}{l}0.03 \\
0.03 \\
0.02 \\
0.03\end{array}$ & $\begin{array}{l}<0.02-0.14 \\
<0.02-0.08 \\
<0.02-0.06 \\
<0.02-0.14\end{array}$ & $\begin{array}{l}69 \\
17 \\
25 \\
27\end{array}$ & $\begin{array}{l}0.04 \\
0.04 \\
0.04 \\
0.03\end{array}$ & $\begin{array}{l}- \\
- \\
- \\
-\end{array}$ & $\begin{array}{l}0.04 \\
0.03 \\
0.03 \\
0.05\end{array}$ & $\begin{array}{l}0.02 \\
0.03 \\
0.02 \\
0.01\end{array}$ & $\begin{array}{l}<0.02-0.07 \\
<0.02-0.07 \\
<0.02-0.07 \\
<0.02-0.07\end{array}$ \\
\hline $\mathrm{Cu}$ & $\begin{array}{l}\text { Overall } \\
\text { Juvenile } \\
\text { First-year } \\
\text { Adult }\end{array}$ & $\begin{array}{l}70 \\
17 \\
27 \\
26\end{array}$ & $\begin{array}{l}4.46 \\
5.20 \\
4.31 \\
4.05\end{array}$ & $\begin{array}{l}4.55 \\
5.37 \\
4.49 \\
4.14\end{array}$ & $\begin{array}{l}4.69 \\
5.42 \\
4.67 \\
4.24\end{array}$ & $\begin{array}{l}1.23 \\
0.82 \\
1.48 \\
0.95\end{array}$ & $\begin{array}{l}2.57-9.10 \\
3.89-6.90 \\
3.01-9.10 \\
2.57-5.87\end{array}$ & $\begin{array}{l}69 \\
17 \\
25 \\
27\end{array}$ & $\begin{array}{l}3.41 \\
3.99 \\
3.00 \\
3.17\end{array}$ & $\begin{array}{l}3.46 \\
4.24 \\
3.19 \\
3.28\end{array}$ & $\begin{array}{l}3.54 \\
4.31 \\
3.26 \\
3.32\end{array}$ & $\begin{array}{l}0.81 \\
0.85 \\
0.70 \\
0.55\end{array}$ & $\begin{array}{l}2.38-6.50 \\
3.41-6.50 \\
2.38-4.80 \\
2.50-4.50\end{array}$ \\
\hline $\mathrm{Fe}$ & $\begin{array}{l}\text { Overall } \\
\text { Juvenile } \\
\text { First-year } \\
\text { Adut }\end{array}$ & $\begin{array}{l}70 \\
17 \\
27 \\
26\end{array}$ & $\begin{array}{l}460 \\
500 \\
466 \\
450\end{array}$ & $\begin{array}{l}463 \\
516 \\
453 \\
441\end{array}$ & $\begin{array}{l}480 \\
543 \\
464 \\
456\end{array}$ & $\begin{array}{r}129 \\
177 \\
97 \\
113\end{array}$ & $\begin{array}{l}208-805 \\
315-805 \\
208-654 \\
231-715\end{array}$ & $\begin{array}{l}69 \\
17 \\
25 \\
27\end{array}$ & $\begin{array}{l}184 \\
175 \\
183 \\
192\end{array}$ & $\begin{array}{l}182 \\
172 \\
179 \\
191\end{array}$ & $\begin{array}{l}184 \\
176 \\
181 \\
193\end{array}$ & $\begin{array}{l}30 \\
38 \\
26 \\
27\end{array}$ & $\begin{array}{l}114-254 \\
114-254 \\
124-223 \\
145-254\end{array}$ \\
\hline $\mathrm{Mg}$ & $\begin{array}{l}\text { Overall } \\
\text { Juvenile } \\
\text { First-yeat } \\
\text { Adult }\end{array}$ & $\begin{array}{l}70 \\
17 \\
27 \\
26\end{array}$ & $\begin{array}{l}208 \\
197 \\
218 \\
198\end{array}$ & $\begin{array}{l}214 \\
220 \\
222 \\
203\end{array}$ & $\begin{array}{l}218 \\
225 \\
224 \\
206\end{array}$ & $\begin{array}{l}43 \\
52 \\
39 \\
40\end{array}$ & $\begin{array}{l}161-365 \\
177-365 \\
176-325 \\
161-295\end{array}$ & $\begin{array}{l}69 \\
17 \\
25 \\
27\end{array}$ & $\begin{array}{l}207 \\
219 \\
202 \\
208\end{array}$ & $\begin{array}{l}217 \\
229 \\
212 \\
215\end{array}$ & $\begin{array}{l}220 \\
232 \\
21.4 \\
21.7\end{array}$ & $\begin{array}{l}35 \\
43 \\
30 \\
33\end{array}$ & $\begin{array}{l}176-365 \\
201-365 \\
176-300 \\
176-295\end{array}$ \\
\hline $\mathrm{Mn}$ & $\begin{array}{l}\text { Overall } \\
\text { Juvenile } \\
\text { First-year } \\
\text { Adult }\end{array}$ & $\begin{array}{l}70 \\
17 \\
27 \\
26\end{array}$ & $\begin{array}{l}2.57 \\
2.81 \\
2.44 \\
2.47\end{array}$ & $\begin{array}{l}2.46 \\
2.79 \\
2.38 \\
2.34\end{array}$ & $\begin{array}{l}2.51 \\
2.82 \\
2.41 \\
2.40\end{array}$ & $\begin{array}{l}0.47 \\
0.42 \\
0.40 \\
0.50\end{array}$ & $\begin{array}{l}1.38-3.50 \\
1.88-3.50 \\
1.78-3.30 \\
1.38-3.23\end{array}$ & $\begin{array}{l}69 \\
17 \\
25 \\
27\end{array}$ & $\begin{array}{l}2.09 \\
2.31 \\
1.96 \\
2.05\end{array}$ & $\begin{array}{l}2.10 \\
2.39 \\
1.95 \\
2.07\end{array}$ & $\begin{array}{l}2.13 \\
2.42 \\
1.97 \\
2.09\end{array}$ & $\begin{array}{l}0.37 \\
0.38 \\
0.32 \\
0.30\end{array}$ & $\begin{array}{l}1.41-3.30 \\
1.84-3.30 \\
1.41-2.57 \\
1.50-2.60\end{array}$ \\
\hline $\mathrm{Pb}$ & $\begin{array}{l}\text { Overall } \\
\text { Juvenile } \\
\text { First-year } \\
\text { Adult }\end{array}$ & $\begin{array}{l}70 \\
17 \\
27 \\
26\end{array}$ & $\begin{array}{l}0.03 \\
0.05 \\
0.04 \\
0.00\end{array}$ & $\begin{array}{l}- \\
- \\
- \\
-\end{array}$ & $\begin{array}{l}0.04 \\
0.06 \\
0.05 \\
0.02\end{array}$ & $\begin{array}{l}0.06 \\
0.06 \\
0.08 \\
0.04\end{array}$ & $\begin{array}{l}<0.06-0.41 \\
<0.06-0.24 \\
<0.06-0.41 \\
<0.06-0.20\end{array}$ & $\begin{array}{l}69 \\
17 \\
25 \\
27\end{array}$ & $\begin{array}{l}0.08 \\
0.09 \\
0.05 \\
0.09\end{array}$ & $\begin{array}{l}- \\
- \\
- \\
-\end{array}$ & $\begin{array}{l}0.10 \\
0.12 \\
0.10 \\
0.10\end{array}$ & $\begin{array}{l}0.11 \\
0.13 \\
0.13 \\
0.08\end{array}$ & $\begin{array}{l}<0.07-0.63 \\
<0.07-0.42 \\
<0.07-0.63 \\
<0.07-0.38\end{array}$ \\
\hline Zn & $\begin{array}{l}\text { Overall } \\
\text { Juvenile } \\
\text { First-year } \\
\text { Adult }\end{array}$ & $\begin{array}{l}70 \\
17 \\
27 \\
26\end{array}$ & $\begin{array}{l}25 \\
27 \\
25 \\
24\end{array}$ & $\begin{array}{l}25 \\
27 \\
26 \\
24\end{array}$ & $\begin{array}{l}25 \\
27 \\
26 \\
24\end{array}$ & $\begin{array}{l}3 \\
3 \\
2 \\
4\end{array}$ & $\begin{array}{l}18-34 \\
21-32 \\
19-32 \\
18-34\end{array}$ & $\begin{array}{l}69 \\
17 \\
25 \\
27\end{array}$ & $\begin{array}{l}20 \\
20 \\
19 \\
21\end{array}$ & $\begin{array}{l}20 \\
21 \\
19 \\
20\end{array}$ & $\begin{array}{l}20 \\
21 \\
19 \\
20\end{array}$ & $\begin{array}{l}2 \\
2 \\
2 \\
2\end{array}$ & $\begin{array}{l}16-25 \\
18-25 \\
17-23 \\
16-25\end{array}$ \\
\hline \multicolumn{14}{|l|}{$\begin{array}{l}\text { Curlew } \\
\text { sandpiper }\end{array}$} \\
\hline $\mathrm{Ca}$ & $\begin{array}{l}\text { Overall } \\
\text { Juvenile } \\
\text { Adult }\end{array}$ & $\begin{array}{l}28 \\
12 \\
16\end{array}$ & $\begin{array}{l}49 \\
54 \\
46\end{array}$ & $\begin{array}{l}54 \\
52 \\
57\end{array}$ & $\begin{array}{r}96 \\
53 \\
128\end{array}$ & $\begin{array}{r}246 \\
12 \\
326\end{array}$ & $\begin{array}{l}28-1350 \\
38-76 \\
28-1350\end{array}$ & $\begin{array}{l}28 \\
12 \\
16\end{array}$ & $\begin{array}{l}54 \\
73 \\
54\end{array}$ & $\begin{array}{l}74 \\
86 \\
66\end{array}$ & $\begin{array}{l}149 \\
156 \\
144\end{array}$ & $\begin{array}{l}329 \\
295 \\
362\end{array}$ & $\begin{array}{l}40-1500 \\
47-1090 \\
40-1500\end{array}$ \\
\hline $\mathrm{Cd}$ & $\begin{array}{l}\text { Overall } \\
\text { Juvenile } \\
\text { Adult }\end{array}$ & $\begin{array}{l}28 \\
12 \\
16\end{array}$ & $\begin{array}{l}0.34 \\
0.04 \\
0.51\end{array}$ & $\begin{array}{l}- \\
\overline{0.80}\end{array}$ & $\begin{array}{l}0.76 \\
0.10 \\
1.26\end{array}$ & $\begin{array}{l}1.14 \\
0.11 \\
1.31\end{array}$ & $\begin{array}{r}<0.06-4.29 \\
<0.06-0.32 \\
0.19-4.29\end{array}$ & $\begin{array}{l}28 \\
12 \\
16\end{array}$ & $\begin{array}{l}0.87 \\
0.12 \\
1.88\end{array}$ & $\begin{array}{l}0.66 \\
0.12 \\
2.33\end{array}$ & $\begin{array}{l}2.21 \\
0.13 \\
3.76\end{array}$ & $\begin{array}{l}3.37 \\
0.05 \\
3.30\end{array}$ & $\begin{array}{l}0.06-14.1 \\
0.06-0.22 \\
0.40-14.1\end{array}$ \\
\hline $\mathrm{Co}$ & $\begin{array}{l}\text { Overall } \\
\text { Juvenile } \\
\text { Adult }\end{array}$ & $\begin{array}{l}28 \\
12 \\
16\end{array}$ & $\begin{array}{l}0.05 \\
0.04 \\
0.06\end{array}$ & $\begin{array}{c}- \\
0.05 \\
-\end{array}$ & $\begin{array}{l}0.05 \\
0.05 \\
0.06\end{array}$ & $\begin{array}{l}0.02 \\
0.01 \\
0.03\end{array}$ & $\begin{array}{r}<0.02-0.10 \\
0.03-0.07 \\
<0.02-0.10\end{array}$ & $\begin{array}{l}28 \\
12 \\
16\end{array}$ & $\begin{array}{l}0.08 \\
0.09 \\
0.08\end{array}$ & $\begin{array}{l}0.08 \\
0.09 \\
0.08\end{array}$ & $\begin{array}{l}0.09 \\
0.09 \\
0.09\end{array}$ & $\begin{array}{l}0.03 \\
0.02 \\
0.04\end{array}$ & $\begin{array}{l}0.04-0.20 \\
0.05-0.12 \\
0.04-0.20\end{array}$ \\
\hline $\mathrm{Cu}$ & $\begin{array}{l}\text { Overall } \\
\text { Juvenile } \\
\text { Adult }\end{array}$ & $\begin{array}{l}28 \\
12 \\
16\end{array}$ & $\begin{array}{l}5.28 \\
6.25 \\
5.00\end{array}$ & $\begin{array}{l}5.62 \\
6.20 \\
5.23\end{array}$ & $\begin{array}{l}5.83 \\
6.44 \\
5.37\end{array}$ & $\begin{array}{l}1.62 \\
1.82 \\
1.34\end{array}$ & $\begin{array}{l}3.68-9.40 \\
3.68-9.40 \\
3.70-8.70\end{array}$ & $\begin{array}{l}28 \\
12 \\
16\end{array}$ & $\begin{array}{l}4.35 \\
4.81 \\
3.84\end{array}$ & $\begin{array}{l}4.35 \\
4.88 \\
3.99\end{array}$ & $\begin{array}{l}4.42 \\
4.92 \\
4.05\end{array}$ & $\begin{array}{l}0.82 \\
0.68 \\
0.72\end{array}$ & $\begin{array}{l}3.06-6.10 \\
3.97-6.10 \\
3.06-5.80\end{array}$ \\
\hline $\mathrm{Fe}$ & $\begin{array}{l}\text { Overall } \\
\text { Juvenile } \\
\text { Adult }\end{array}$ & $\begin{array}{l}28 \\
12 \\
16\end{array}$ & $\begin{array}{l}542 \\
674 \\
509\end{array}$ & $\begin{array}{l}550 \\
643 \\
489\end{array}$ & $\begin{array}{l}565 \\
652 \\
500\end{array}$ & $\begin{array}{l}131 \\
111 \\
106\end{array}$ & $\begin{array}{l}340-830 \\
433-830 \\
340-705\end{array}$ & $\begin{array}{l}28 \\
12 \\
16\end{array}$ & $\begin{array}{l}174 \\
193 \\
169\end{array}$ & $\begin{array}{l}173 \\
186 \\
164\end{array}$ & $\begin{array}{l}176 \\
188 \\
167\end{array}$ & $\begin{array}{l}30 \\
28 \\
28\end{array}$ & $\begin{array}{l}116-240 \\
145-240 \\
116-210\end{array}$ \\
\hline $\mathrm{Mg}$ & $\begin{array}{l}\text { Overall } \\
\text { Juvenile } \\
\text { Adult }\end{array}$ & $\begin{array}{l}28 \\
12 \\
16\end{array}$ & $\begin{array}{l}218 \\
199 \\
220\end{array}$ & $\begin{array}{l}237 \\
243 \\
232\end{array}$ & $\begin{array}{l}247 \\
256 \\
240\end{array}$ & $\begin{array}{l}76 \\
87 \\
70\end{array}$ & $\begin{array}{l}175-435 \\
175-385 \\
177-435\end{array}$ & $\begin{array}{l}28 \\
12 \\
16\end{array}$ & $\begin{array}{l}214 \\
214 \\
213\end{array}$ & $\begin{array}{l}225 \\
235 \\
217\end{array}$ & $\begin{array}{l}227 \\
239 \\
218\end{array}$ & $\begin{array}{l}33 \\
42 \\
21\end{array}$ & $\begin{array}{l}193-305 \\
196-305 \\
193-270\end{array}$ \\
\hline $\mathrm{Mn}$ & $\begin{array}{l}\text { Overall } \\
\text { Juvenile } \\
\text { Adult }\end{array}$ & $\begin{array}{l}28 \\
12 \\
16\end{array}$ & $\begin{array}{l}2.70 \\
2.60 \\
2.76\end{array}$ & $\begin{array}{l}2.67 \\
2.69 \\
2.65\end{array}$ & $\begin{array}{l}2.73 \\
2.75 \\
2.70\end{array}$ & $\begin{array}{l}0.61 \\
0.67 \\
0.58\end{array}$ & $\begin{array}{l}1.92-4.40 \\
1.92-4.40 \\
1.97-4.40\end{array}$ & $\begin{array}{l}28 \\
12 \\
16\end{array}$ & $\begin{array}{l}2.08 \\
2.05 \\
2.11\end{array}$ & $\begin{array}{l}2.02 \\
2.00 \\
2.04\end{array}$ & $\begin{array}{l}2.04 \\
2.02 \\
2.06\end{array}$ & $\begin{array}{l}0.26 \\
0.25 \\
0.26\end{array}$ & $\begin{array}{l}1.60-2.45 \\
1.60-2.40 \\
1.60-2.45\end{array}$ \\
\hline $\mathrm{Pb}$ & $\begin{array}{l}\text { Overall } \\
\text { Juvenile } \\
\text { Adult }\end{array}$ & $\begin{array}{l}28 \\
12 \\
16\end{array}$ & $\begin{array}{l}0.04 \\
0.07 \\
0.03\end{array}$ & $\begin{array}{l}- \\
- \\
-\end{array}$ & $\begin{array}{l}0.06 \\
0.08 \\
0.05\end{array}$ & $\begin{array}{l}0.07 \\
0.06 \\
0.07\end{array}$ & $\begin{array}{l}<0.06-0.23 \\
<0.06-0.19 \\
<0.06-0.23\end{array}$ & $\begin{array}{l}28 \\
12 \\
16\end{array}$ & $\begin{array}{l}0.05 \\
0.10 \\
0.00\end{array}$ & $\begin{array}{l}- \\
- \\
-\end{array}$ & $\begin{array}{l}0.06 \\
0.11 \\
0.03\end{array}$ & $\begin{array}{l}0.06 \\
0.05 \\
0.04\end{array}$ & $\begin{array}{l}<0.07-0.20 \\
<0.07-0.20 \\
<0.07-0.12\end{array}$ \\
\hline $\mathrm{Zn}$ & $\begin{array}{l}\text { Overall } \\
\text { Juvenile } \\
\text { Adult }\end{array}$ & $\begin{array}{l}28 \\
12 \\
16\end{array}$ & $\begin{array}{l}24 \\
25 \\
23\end{array}$ & $\begin{array}{l}25 \\
25 \\
24\end{array}$ & $\begin{array}{l}25 \\
25 \\
25\end{array}$ & $\begin{array}{l}5 \\
3 \\
6\end{array}$ & $\begin{array}{l}17-41 \\
21-29 \\
17-41\end{array}$ & $\begin{array}{l}28 \\
12 \\
16\end{array}$ & $\begin{array}{l}21 \\
21 \\
21\end{array}$ & $\begin{array}{l}21 \\
21 \\
21\end{array}$ & $\begin{array}{l}21 \\
21 \\
21\end{array}$ & $\begin{array}{l}2 \\
2 \\
2\end{array}$ & $\begin{array}{l}18-25 \\
18-24 \\
18-25\end{array}$ \\
\hline
\end{tabular}


Table 3. Calidris alpina and C. feruginea. Statistically significant inter-element concentration associations in liver (upper right) and in kidney tissue (lower left) as revealed by Kendall rank-order correlation coefficient ( $\tau$ ). Upper row for each metal: coefficients for dunlin; lower row: coefficients for curlew sandpiper Co-occurrences of a significant coefficient in both species are in bold type. $\mathrm{n}$ as in Table 2 . Significance levels: ${ }^{\prime} \mathrm{p}<0.05 ;{ }^{\cdots} \mathrm{p}<0.01{ }^{\prime} \cdots p<0.001$

\begin{tabular}{|c|c|c|c|c|c|c|c|c|}
\hline \multicolumn{9}{|c|}{ Liver } \\
\hline & $\mathrm{Ca}$ & $\mathrm{Cd}$ & $\mathrm{Cu}$ & $\mathrm{Fe}$ & $\mathrm{Mg}$ & $\mathrm{Mn}$ & $\mathrm{Zn}$ & \\
\hline $\mathrm{Ca}$ & & $\begin{array}{c}-0.24 \\
-\end{array}$ & $\begin{array}{c}0.26^{\circ} \\
-\end{array}$ & $\begin{array}{c}0.31 \\
-\end{array}$ & - & - & $\begin{array}{c}0.38 \cdots \\
-\end{array}$ & $\mathrm{Ca}$ \\
\hline $\mathrm{Cd}$ & $\begin{array}{c}-0.18^{\circ} \\
-\end{array}$ & & - & - & - & - & - & $\mathrm{Cd}$ \\
\hline $\mathrm{Cu}$ & - & $\begin{array}{c}-0.21^{\circ} \\
-\end{array}$ & & $\begin{array}{l}0.23^{\circ} \\
0.33^{\circ}\end{array}$ & $\begin{array}{l}0.41^{\cdots} \cdots \\
0.46^{\prime} \cdots\end{array}$ & $\begin{array}{l}0.42^{\cdots} \\
0.27^{\circ}\end{array}$ & $\begin{array}{l}0.33^{\cdots} \cdots \\
0.43 \cdots\end{array}$ & $\mathrm{Cu}$ \\
\hline $\mathrm{Fe}$ & $\begin{array}{c}0.19^{\circ} \\
-\end{array}$ & - & - & & $\begin{array}{c}0.17^{\circ} \\
-\end{array}$ & - & $\begin{array}{c}0.32 \cdots \\
-\end{array}$ & $\mathrm{Fe}$ \\
\hline Mg & $0.23 \cdots$ & - & $\begin{array}{l}0.54 \cdots \\
0.39 \cdots\end{array}$ & - & & $\begin{array}{c}0.38 \cdots \\
-\end{array}$ & - & $\mathrm{Mg}$ \\
\hline $\mathrm{Mn}$ & $\begin{array}{c}0.19^{\circ} \\
-\end{array}$ & $\begin{array}{c}-0.20^{\circ} \\
-\end{array}$ & $0.35 \cdots$ & - & $\begin{array}{c}0.23^{\circ} \\
-\end{array}$ & & $\begin{array}{c}0.18^{\circ} \\
-\end{array}$ & $\mathrm{Mn}$ \\
\hline \multirow[t]{2}{*}{ Zn } & $\begin{array}{c}0.34 \cdots \\
-\end{array}$ & $\begin{array}{c}0.17^{\circ} \\
-\end{array}$ & - & - & -0.34. & $\begin{array}{c}0.30^{\cdots} \\
-\end{array}$ & & $\mathrm{Zn}$ \\
\hline & $\mathrm{Ca}$ & $\mathrm{Cd}$ & $\mathrm{Cu}$ & $\begin{array}{c}F e \\
\text { Kidney }\end{array}$ & $\mathrm{Mg}$ & $\mathrm{Mn}$ & $\mathrm{Zn}$ & \\
\hline
\end{tabular}

Table 4. Calidris alpina and C. ferruginea. Statistically significant element concentration associations in kidney versus liver tissue as revealed by Kendall rank-order correlation coefficient $(\tau)$. Upper row for each metal: coefficients for dunlin; lower row: coefficients for curlew sandpiper. Co-occurrences of a significant coefficient in both species are in bold type. $\mathrm{n}$ as in Table 2 . Significance levels: " $p<0.05 ; \cdots p<0.01 ; \cdots p<0.001$

\begin{tabular}{|c|c|c|c|c|c|c|c|}
\hline \multirow{2}{*}{$\begin{array}{l}\text { Metal in } \\
\text { kidney }\end{array}$} & \multicolumn{7}{|c|}{ Metal in liver } \\
\hline & $\mathrm{Ca}$ & $\mathrm{Cd}$ & $\mathrm{Cu}$ & $\mathrm{Fe}$ & $\mathrm{Mg}$ & $\mathrm{Mn}$ & $\mathrm{Zn}$ \\
\hline \multirow{2}{*}{$\mathrm{Ca}$} & $0.18^{\circ}$ & $-0.25^{\prime}$ & - & - & - & - & - \\
\hline & - & $-0.29^{\circ}$ & $-0.35^{\circ}$ & - & - & $-0.29^{\circ}$ & - \\
\hline \multirow{2}{*}{$\mathrm{Cd}$} & $-0.29 \cdots$ & $0.70 \cdots$ & $-0.22 \cdots$ & $-0.19^{\circ}$ & - & $-0.21^{\circ}$ & $-0.17^{\circ}$ \\
\hline & - & $0.82 \cdots$ & - & $-0.30^{\circ}$ & - & - & - \\
\hline \multirow{2}{*}{$\mathrm{Cu}$} & - & - & $0.50^{\cdots}$ & - & $0.27^{\circ}$ & $0.38 \cdots$ & - \\
\hline & - & - & $0.51 \cdots$ & - & - & - & - \\
\hline \multirow{2}{*}{$\mathrm{Fe}$} & - & - & - & $0.21^{\circ}$ & - & $-0.20^{\circ}$ & - \\
\hline & - & - & - & - & - & - & - \\
\hline \multirow{2}{*}{$\mathrm{Mg}$} & - & - & $0.37^{\cdots}$ & - & $0.32 \cdots$ & $0.31 \cdots$ & - \\
\hline & - & - & $0.31^{\circ}$ & - & $0.48 \cdots$ & - & - \\
\hline \multirow{2}{*}{$\mathrm{Mn}$} & - & $-0.18^{\circ}$ & $0.20^{\circ}$ & - & - & $0.40^{\circ} \cdots$ & - \\
\hline & - & - & - & - & - & $0.34^{\bullet}$ & - \\
\hline \multirow{2}{*}{$\mathrm{Zn}$} & - & - & - & - & $-0.32 \cdots$ & - & - \\
\hline & - & - & - & - & $-0.29^{\circ}$ & - & - \\
\hline
\end{tabular}



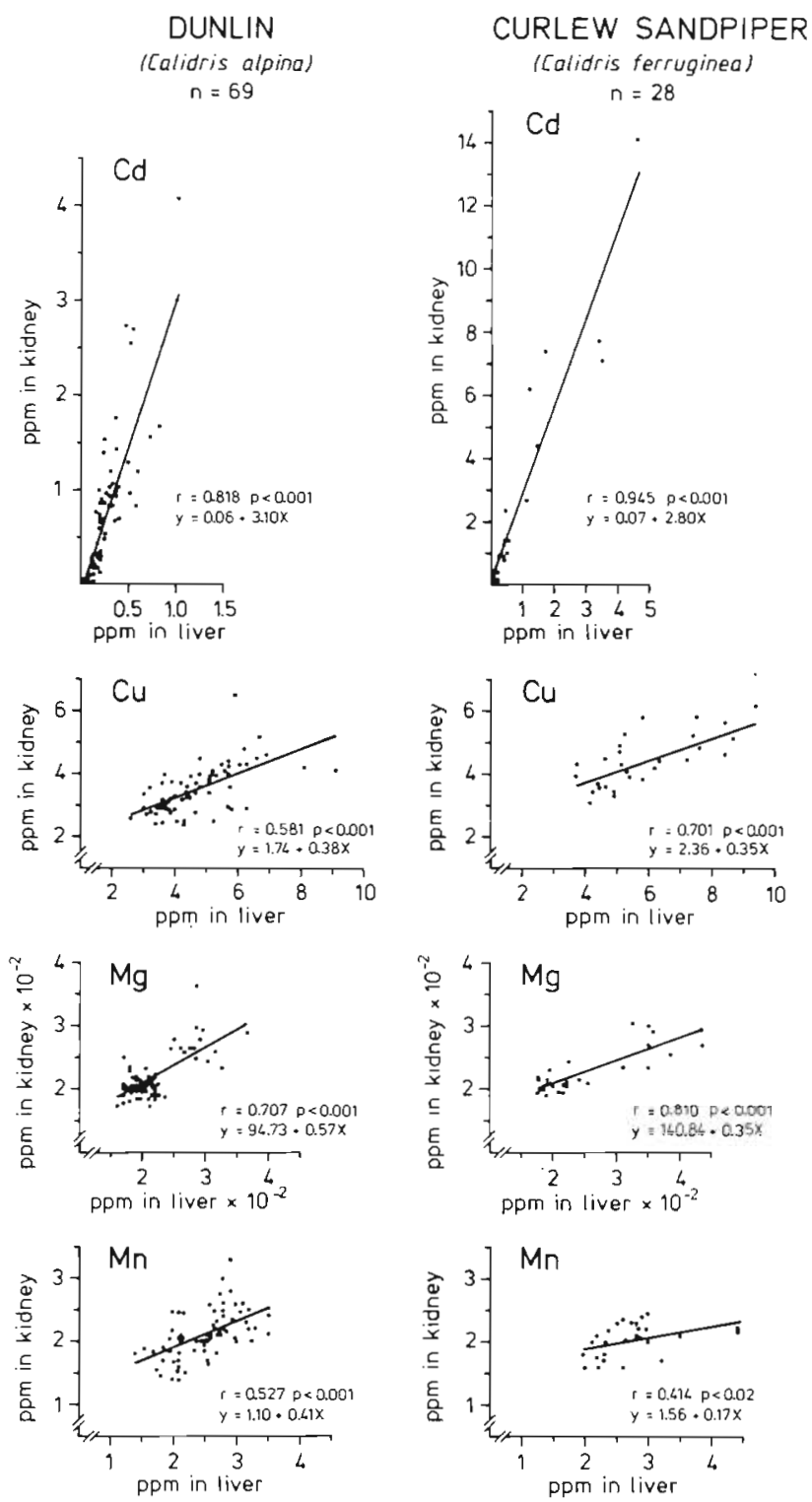

Fig. 2. Calidris alpina and C. ferruginea. Linear regressions of cadmium, copper, magnesium and manganese concentrations in kidney versus liver tissues (fresh wt) of dunlin and curlew sandpiper. $r$ indicates Pearson product-moment correlation coefficient. Note different scales of the axes

Thus, in both cases the relative accumulation is higher in curlew sandpiper than in dunlin. The more rightskewed (positively skewed) distribution patterns of curlew sandpiper, reflected in relatively larger discrepancies between the arithmetic means and corresponding geometric means or medians (Table 2), indicates overcalculation of these numerical differences. However, when comparison of the 2 latter, less distribution-biased estimates is possible, the same tendency is revealed. Demographic differences in the collected populations, as well as differences in cadmium exposure via food, are both plausible explana-

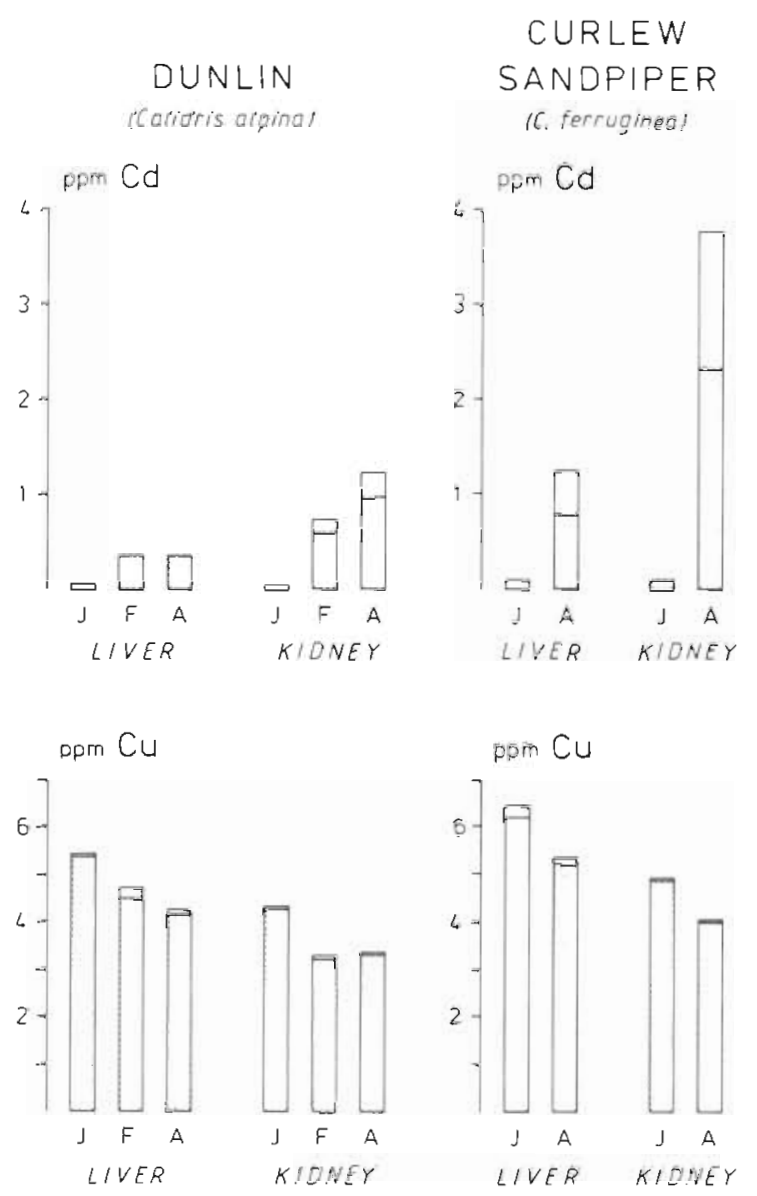

Fig. 3. Calidris alpina and C. ferruginea. Age-related concentration changes of cadmium and copper in liver and kidney tissues (fresh wt) of dunlin and curlew sandpiper, expressed as geometric mean (stippled part of columns) and arithmetic mean (open part). Age classes: $J=$ juveniles, $F=$ first-year birds, $\mathrm{A}=$ adults. Note different scales of axes

tions for the interspecific disparities found. In the dunlin, this age-related accumulation is already significant in the passing first-year birds, with an average enrichment factor of $5.5(p<0.001)$ for the liver and 10.3 ( $\mathrm{p}<0.001$ ) for the kidney tissues, compared to juveniles. A subsequent age-related concentration increase between first-year birds and adults was not observable in the liver tissue of our dunlins, but in the kidney tissue the recorded 1.7 -fold increase is statistically significant $(p<0.02)$. An increase with age of cadmium concentration in liver and kidney tissues has previously been documented for a variety of birds and mammals (e.g. Sabbioni et al. 1978, Frank \& Borg 1979, Furness \& Hutton 1979, Cherry 1981, Hutton 1981, Hamanaka et al. 1982, Maedgen et al. 1982, Ronald et al. 1984, Elinder 1985).

Overall, the cadmium concentrations in the kidney versus the liver tissues of dunlin or curlew sandpiper is strongly, linearly correlated (Fig. 2). The regression 
coefficients of 3.10 in dunlins and 2.80 in curlew sandpipers compare fairly well with renal to hepatic ratios in mallards Anas platyrhynchos (range: 2.16 to 3.18 ) fed ad libitum a low or moderately (2 to $20 \mathrm{ppm} \mathrm{Cd}$ ) treated diet (calculated from data in White \& Finley 1978), and also with a reported linear regression coefficient of 3.6 in the herring gull Larus argentatus (Nicholson 1981), but are lower than a linear regression coefficient of 10.34 in the great skua Stercorarius skua (calculated from data in Furness \& Hutton 1979). A corresponding coefficient of 8.04 may be calculated from a study of the Steller sea lion Eumetopias jubata (Hamanaka et al. 1982). In humans, the renal to hepatic ratio of cadmium changes with age, within a normal range of between 3 and 30 (Kjellström 1979, Elinder 1985; recalculated by us to whole kidney concentration according to Svartengren et al. 1986).

\section{Biological half-life}

If we operationally assume that the renal absorption of cadmium is continual and constant and its ratio with the elimination rate is also relatively constant, a first order estimate of the mean biological half-life of this element in the sampled dunlin kidney tissue can be calculated via the equation:

$$
\mathrm{Cd}=\mathrm{A}\left(1-\mathrm{e}^{-\mathrm{bt}}\right)
$$

where $\mathrm{Cd}=$ accumulated cadmium concentration; $\mathrm{A}$ $=$ saturation constant at steady state (adsorption rate equals elimination rate) $; b=$ elimination constant; $t=$ time since exposure started (age).

The biological half-life $\left(t_{1 / 2}\right)$ can then be determined accordingly:

$$
\mathrm{t}_{1 / 2}=\ln 2 / \mathrm{b}
$$

This method of estimating the mean biological half-life of cadmium (adapted from Tsuchiya \& Sugita 1971, see also Task Group on Metal Accumulation 1973) is a close parallel to related calculations in radionuclide chemistry (e.g. Siri 1949, Choppin \& Rydberg 1980).

In the present study we know only that the adult class of the dunlins consists of birds of $2.1 \mathrm{yr}$ old and older. However, a demographic life table calculation (Farner 1949) and an assumption of a steady rate of mortality independent of age (which seems reasonable for adult birds, see e.g. Farner 1955, Cody 1971, Murton \& Westwood 1977), provides an opportunity to calculate an estimate of the adult age class via the formula:

$$
E_{\mathrm{x}}=100 / \mathrm{q}_{\mathrm{x}}-0.5
$$

where $E_{x}=$ mean life expectancy; $q_{x}=$ average annual mortality rate $(\%)$.
Based on a detailed breeding study of a Finnish dunlin population (C. alpina schinizii) Soikkeli (1970) estimated $\mathrm{q}_{\mathrm{x}}$ to $25 \%$ in adults, which he reckoned to be an overestimate. If we adopt this figure, a mean age of 5.6 yr $[(100 / 25-0.5)+2.1=5.6]$ is obtained for the adult class of dunlin in the present study. By using a lower $q_{x}$ value of $20 \%$, a corresponding mean age of 6.6 yr is obtained.

Age accumulation regressions (fitted by the iterative method and chosen on the basis of revealed least residual variance) of Equation 1 were obtained (Fig. 4)

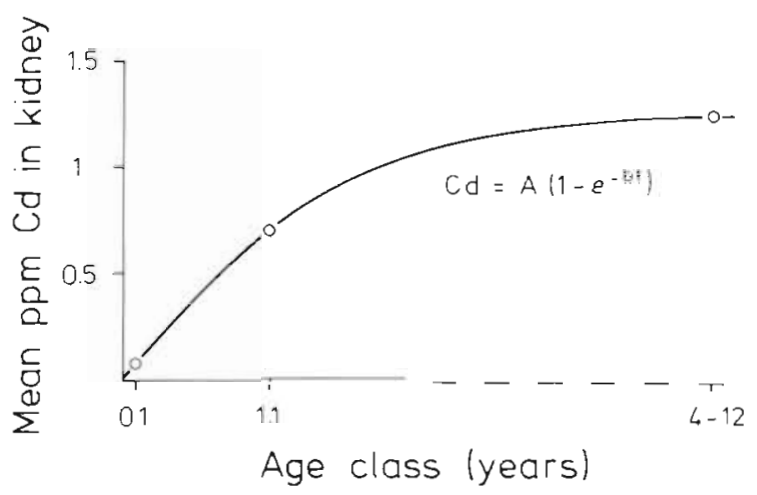

Fig. 4. Calidris alpina. Principle age accumulation curve of renal cadmium concentration (arithmetic mean of fresh wt) versus age class of dunlin sampled. For details of equation given, see text

from primary renal concentration data of the juvenile and first year birds and assigned values from 4 to $12 \mathrm{yr}$ as the average age of our adult specimens combined with an assigned value of either (1) the upper $95 \%$ confidence limit (2.83 ppm), (2) the upper one standard deviation $(2.15 \mathrm{ppm})$, or (3) the arithmetic mean $(1.23 \mathrm{ppm})$ as the cadmium concentration of this age class. Obtained $b$ values have been calculated to biological half-life by Equation 2, and illustrated in Fig. 5.

The upper curve in this figure however, is most likely an overestimate of the renal biological half-life of the sampled birds since only one primary value falls above the $95 \%$ confidence limit. If we instead regard the arithmetic mean and one standard deviation as the rational upper demarcation, and $6 y \mathrm{y}$ as a mean age of the sampled adults, it is justifiable to infer that the mean biological half-life in the kidney tissue of the dunlin is about 1 yr, but below $2.5 \mathrm{yr}$.

The magnitude of this half-life estimate is of interest. To our knowledge, this is the first time this crucial toxicological parameter has been assessed for cadmium in birds or any wild animal population. Our estimate corresponds approximately to calculations of renal biological half-lifes of 433 and $990 \mathrm{~d}$, determined 


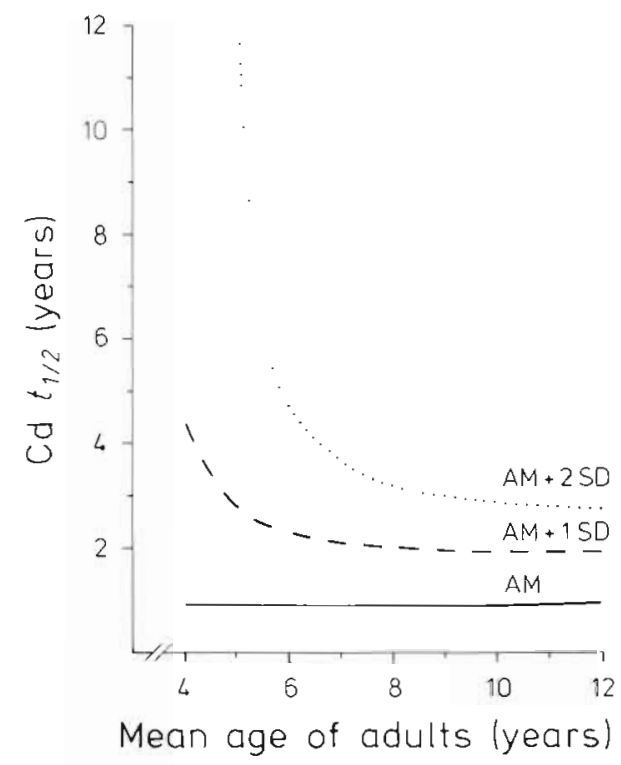

Fig. 5. Calidris alpina. Modelled graphs of relation between biological half-life $\left(t_{1 / 2}\right)$ of renal cãdmium versus mean age of adult class of dunlin sampled. Three renal concentration values (fresh wt) of adult birds are considered: (-) arithmetic mean (AM); (---) AM + 1 standard deviation (SD); ( . .) AM +2 SD, i.e. the upper $95 \%$ confidence limit

by ${ }^{109} \mathrm{CdCl}_{2}$ administrated orally and injected subcutaneously, respectively, in ICR mice (MatsubaraKhan 1974), but is lower than estimates on human kidney tissue ranging from 6 to 38 yr (Kjellström \& Nordberg 1978).

\section{CONCLUSIONS}

The present paper shows that the levels of the 10 metals determined in liver and kidney tissues of dunlins and curlew sandpipers, passing the Baltic Sea on southward migration in the autumn, are within the ranges normally found in birds and mammals (e.g. Underwood 1977, Eisler 1981, Bryan 1984, Nyholm 1985). For metals with rapid physiological depuration or efficient homeostatic regulation the loadings of the birds might, however, have been reduced during the time on the breeding grounds, and thus might have been higher in spring than recorded in the present study (cf. Parslow 1973, Evans \& Moon 1981).

For cadmium, the concentration in the organs increases with age, whereas the opposite is true for copper. The highest renal cadmium concentration recorded in an adult curlew sandpiper however, (14.1 ppm), is still lower than the concentrations of 25 to $50 \mathrm{mg}$ per $\mathrm{kg}$ wet tissue reported for nephrotoxic lesions in birds (White et al. 1978, Nicholson \& Osborn 1983, Nicholson et al. 1983; in some instances recalcu- lated by us to wet weight basis by the divisor 4 , according to Scanlon 1982, Karlog et al. 1983, Ohlendorf et al. 1985). However, this is not a rationale or justification for reducing future research effort concerning cadmium in waders, since it seems unwise to restrict the assessment of environmental contaminants on wild animals to histological lesions only. Cadmium has a complex interaction with the metabolism of certain essential elements such as calcium, copper, iron, manganese, selenium and zinc and also with nutrients such as vitamins (e.g. Doyle 1977, Chang et al. 1981, Neathery 1981). Supported by findings in a cadmium ad libitum ingestion experiment on mallard (Di Giulio 1982), Di Giulio \& Scanlon (1985) recently emphasized the importance of considering the potential impact of low level burdens of toxic substances such as cadmium, in combination with recurring physiological stresses such as food shortage and energy drain during migrations. Alterations in energy metabolism during such stresses may be enhanced by the contaminants, and thus become real ecotoxicological hazards. Therefore, consider the following: (1) the relatively long biological half-life in the kidneys tentatively found (Fig. 5) in relation to average life span of the birds, and the marked increase with age (Fig. 3) recorded in the renal cadmium loading (i.e. in the critical organ; sensu Task Group on Metal Accumulation 1973); (2) the insidious and long-term toxic effect of cadmium when accumulated (e.g. Doyle 1977, Chang et al. 1981, Neathery 1981, Elinder 1982); combined with (3) a dramatic increase in anthropogenic mobilization and emission to the environment of this heavy metal during the 20th century (Nriagu 1979); and (4) the physiochemical speciation of cadmium in natural water and sediment with a generally high proportion of soluble or readily desorbed species and thereby high mobility, which is especially valid for brackish and marine conditions (Khalid 1980, Raspor 1980, Simpson 1981, Förstner 1984, Moore \& Ramamoorthy 1984). From these considerations, it seems that cadmium may constitute a future environmental hazard for Calidris sandpipers, as well as for many other aquatic birds. Further studies of the exposure to cadmium and its biological renal half-life in relation to life span, especially in longlived species, are called for.

Acknowledgements. We are grateful to Jan Pettersson of the Ottenby Bird Observatory for the collecting of birds and to Torsten Mörner of the Wildlife Division, National Veterinary Institute, Uppsala, for performing the necropsies. Anders Bignert of the Data Section of the National Swedish Environment Protection Board and Fredrik Granath of the Department of Mathematics of the University of Stockholm kindly assisted us with the statistical computing. We thank Richard $T$. Di Giulio, Carl-Gustaf Elinder, Ragnar Elmgren, Christian Hjort and Bengt-Owe Jansson for reading of drafts and Maureen Moir and John Cayford for linguistic corrections. Birds were 
collected under licence from the National Swedish Environment Protection Board. This study was supported by the Swedish Society for the Conservation of Nature (through a grant from "Valdemar och Emmy Gustafssons naturvårdsfond') and by the National Swedish Environment Protection Board. This is contribution No. 108 from the Ottenby Bird Observatory.

\section{LITERATURE CITED}

Allen Rey, A. (ed.) (1982). SAS user's guide: basics. SAS Inst., Cary, North Carolina

Altenburg, W., Engelmoer, M., Mes, R., Piersma, T. (1982). Wintering waders on the Banc d'Arguin, Mauritania. Stichting Veth tot steun aan Waddenonderzoek, Leiden

Andersson, C. (1985a). Födoval hos en gammal pilgrimsfalkhanne vid Getterön juni-september 1979-1984. Meddelande nr 20 från Getteröns Fågelstation 1984, p. 2-10

Andersson, C. (1985b). Födoval hos genomflyttande och övervintrande pilgrimsfalkar vid Getterön och några andra kustlokaler i mellersta Halland 1937-1984. Meddelande nr 20 frân Getteröns Fågelstation 1984, p. 11-18

Baird, D., Evans, P. R., Milne, H., Pienkowski, M. W. (1985). Utilization by shorebirds of benthic invertebrate production in intertidal areas. Oceanogr. mar. Biol. A. Rev. 23: $573-597$

Blomquist, S. (1985). A bibliographic note on wader publishing. Wader Study Group Bull. 43: 18-20

Bryan, G. W. (1984). Pollution due to heavy metals and their compounds. In: Knne, O. (ed.) Marine ecology, Vol. V, Ocean management, Part 3. Wiley, Chichester, p. 1289-1431

Bub, H. (1971). Vogelfang und Vogelberingung. Teil I. Allgemeines und Fang mit Siebfallen und Reusen. Die Neue Brehm-Bücherei, A. Ziemsen Verlag, Wittenberg

Cade, T. J. (1960). Ecology of the peregrine and gyrfalcon populations in Alaska. Univ. Calif. Publ. Zool. 63; 151-289

Chang, L. W., Reuhl, K. R., Wade, P. R. (1981). Pathological effects of cadmium poisoning. In: Nriagu, J. O. (ed.) Cadmium in the environment. Part II: Health effects. John Wiley and Sons, New York, p. 783-839

Cherry, W. H. (1981). Distribution of cadmium in human tissues. In: Nriagu, J. O. (ed.) Cadmium in the environment. Part II: Health effects. John Wiley and Sons, New York, p. 69-536

Choppin, G. R., Rydberg, J. (1980). Nuclear chemistry, theory and applications. Pergamon Press, Oxford

Clausen, B., Karlog, O., Andersen, A., Elvestad, K., HaldMortensen, P., Andersen-Harild, P. (1985). Total mercury in birds shot near a chemical factory on Harboore Tange, Nordvestjylland. Dansk Vet.-tidsskr. 68: 492-500. (In Danish; English summary)

Clunie, F. (1976). A Fiji peregrine (Falco peregrinus) in an urban-marine environment. Notomis 23: 8-28

Cody, M. L. (1971). Ecological aspects of reproduction. In: Fanner, D. S., King, J. R. (ed.) Avian biology, Vol. 1. Academic Press, New York, p. 461-512

Cramp, S., Simmons, K. E. L. (ed.) (1983). The birds of the Western Palearctic, Vol. 3. Oxford University Press, Oxford

Dejonghe, J. F., Czajkowski, M. A. (1983). Sur la longévité des oiseaux bagués en France métropolitaine, dans les départements d'outre-mer et dans les pays d'influence française. Alauda $51: 27-47$

Dekker, D. (1980). Hunting success rates, foraging habits, and prey selection of peregrine falcons migrating through central Alberta. Can. Field-Nat. 94: 371-382

Dement'ev, G. P., Gladkov, N. A. (ed.) (1969). Birds of the Soviet Union, Vol. 3. Israel Progr. Sci. Transl., Jerusalem

Di Giulio, R. T (1982). The occurrence and toxicology of heavy metals in Chesapeake Bay waterfowl. Ph. D. thesis, Virginia Polytech. Inst. and Virginia State Univ., Blacksburg, Virginia

Di Giulio, R. T., Scanlon, P. F. (1985). Effects of cadmium ingestion and food restriction on energy metabolism and tissue metal concentrations in mallard ducks (Anas platyrhychos). Environ. Res. 37: 433-444

Doyle, J. J. (1977). Effects of low levels of dietary cadmium in animals - a review. J. environ. Qual. 6: 111-116

Duinker, J. C., Hillebrand, M. T. J., Nolting, R. F. (1979). Organochlorines and metals in harbour seals (Dutch Wadden Sea). Mar. Pollut. Bull. 10: 360-364

Edelstam, C. (1972). The visible migration of birds at Ottenby, Sweden. Vå̀r Fågelvärld, Suppl. 7

Eisler, R. (1981). Trace metal concentrations in marine organisms. Pergamon Press, New York

Elinder, C.-G. (1982). Cadmium and health: a survey. Int. J. environ. Studies 19: 187-193

Elinder, C.-G. (1985). Normal values for cadmium in human tissues, blood, and urine in different countries. In: Friberg, L. Elinder, C.-G., Kjellström, T., Nordberg, G. F. (ed.) Cadmium and health: a toxicological and epidemiological appraisal. Vol. 1. Exposure, dose, and metabolism. CRC Press, Boca Raton, Florida, p. 81-102

Elliott, C. C. H., Waltner, M., Underhill, L. G., Pringle, J. S., Dick, W. J. A. (1976). The migration system of the curlew sandpiper Calidris ferruginea in Africa. Ostrich 47: $191-213$

Esmen, N. A., Hammad, Y. Y. (1977). Log-normality of environmental sampling data. J. environ. Sci. Health $12 \mathrm{~A}$ : $29-41$

Evans, P. R., Moon, S. J. (1981). Heavy metals in shorebirds and their prey in north-east England. In: Say, P. J., Whitton, B. A. (ed.) Heavy metals in northern England: environmental and biological aspects. Dept. Botany, Univ. Durham, Durham, p. 181-190

Farner, D. S. (1949). Age groups and longevity in the American robin: comments, further discussion, and certain revisions. Wilson Bull. 61: 68-81

Farner, D. S. (1955). Birdbanding in the study of population dynamics. In: Wolfson, A. (ed.) Recent studies in avian biology. University of Illinois Press, Urbana, Illinois, p. $397-449$

Flint, V. E., Boehme, R. L., Kostin, Y. V., Kuznetsov, A. A. (1984). A field guide to birds of the USSR. Princeton University Press, Princeton. New Jersey

Förstner, U. (1980). Inorganic pollutants, particularly heavy metals in estuaries. In: Olausson, E., Cato, I. (ed.) Chemistry and biogeochemistgry of estuaries. John Wiley \& Sons, Chichester, p. $307-348$

Forstner, U. (1984). Effects of salinity on the metal sorption onto organic particulate matter. Netherl. Inst. Sea Res. Publ. Ser. 10: 195-209

Förstner, U., Müller, G. (1974). Schwermetalle in Flussen und Seen als Ausdruck der Umweltverschmutzung. SpringerVerlag, Berlin

Förstner, U., Wittmann, G. T W. (1981). Metal pollution in the aquatic environment (2nd edn). Springer-Verlag, Berlin

Foumier, O., Dick, W. (1981). Preliminary survey of the Archipel des Bijagos, Guinea-Bissau. Wader Study Group Bull. 31: 24-25

Frank, A. (1976). Automated wet ashing and multi-metal 
determination in biological materials by atomic-absorption spectrometry. Z. analyt. Chem. 279: 101-102

Frank, A., Borg, K. (1979). Heavy metals in tissues of the mute swan (Cygnus olor). Acta Vet. Scand. 20: 447-465

Frank, A., Petersson, L. R. (1983). Selection of operating conditions and analytical procedure in multi-metal analysis of animal tissues by d.c. plasma-atomic emission spectroscopy. Spectrochim. Acta 38 B: 207-220

Furness, R., Hutton, M. (1979). Pollutant levels in the great skua Catharacta skua. Environ. Pollut. 19: 261-268

Gabrielson, I. N., Lincoln, F. C. (1959). The birds of Alaska. The Stackpole Co., Harrisburg, Pennsylvania, and Wildlife Management Inst., Washington, D.C

Glutz von Blotzheim, U. N., Bauer, K. M., Bezzel, E. (1975) Handbuch der Vögel Mitteleuropas. Band 6. Charadriiformes (1. Teil). Akademische Verlagsgesellschaft, Wiesbaden

Goede, A. A. (1985). Mercury, selenium, arsenic and zinc in waders from the Dutch Wadden Sea. Environ. Pollut. 37 A: 287-309

Goede, A. A., de Bruin, M. (1984). The use of bird feather parts as a monitor for metal pollution. Environ. Pollut. 8 B 281-298

Goede, A. A., de Bruin, M. (1985a). Arsenic in the dunlin (Calidris alpina) from the Dutch Waddenzee. Bull. environ. Contam. Toxicol. 34:617-622

Goede, A. A., de Bruin, M. (1985b). Selenium in a shore bird, the dunlin, from the Dutch Waddenzee. Mar. Pollut. Bull. 16: $115-117$

Goede, A. A., de Voogt, P. (1985). Lead and cadmium in waders from the Dutch Wadden Sea. Environ. Pollut. $37 \mathrm{~A}$ $311-322$

Greenwood, J. G. (1984). Migration of dunlin Calidris alpina: a worldwide overview. Ring. Migr. 5: 35-39

Greenwood, J. G. (1986). Geographical variation and taxonomy of the dunlin Calidris alpina (L.). Bull. Brit. Orn. Cl. 106: 43-56

Grimes, L. G. (1974). Radar tracks of Palaeartic waders departing from the coast of Ghana in spring. Ibis 116: 165-171

Hamanaka, T., Itoo, T., Mishima, S. (1982). Age-related change and distribution of cadmium and zinc concentrations in the Steller sea lion (Eumetopias jubata) from the coast of Hokkaido, Japan. Mar. Pollut. Bull. 13: 57-61

Hardy, A. R., Minton, C. D. T. (1980). Dunlin migration in Britain and Ireland. Bird Study 27: 81-92

Hautala, K., Sulkava, S. (1977). Food choice for Finnish peregrines (Falco peregrinus) In: Lindberg, P. (ed.) Pilgrimsfalk. Report from a Peregrine Conference held at Grimsö Wildlife Research Station, Sweden, 1-2 April 1977. Swed. Soc. Conserv. Nat., Stockholm, p. 43-48. (In Swedish; English Summary)

Haviland, M. D. (1915). Notes on the breeding-habits of the curlew-sandpiper. Brit. Birds 8: 178-183

Hildén., O., Hyytiä, K. (1981). The population changes and present status of waders in Finland. In: Runde, $\mathrm{O}$. J., Barrett, R. T. (ed.) Proc. Second Nordic Congr. Ornithol., 1979. Norweg. Orn. Soc., Stavanger, p. 19-37. (In Swedish; English summary)

Hutton, M. (1981). Accumulation of heavy metals and selenium in three seabird species from the United Kingdom. Environ. Pollut. 26 A: 129-145

Johnsgard, P. A. (1981). The plovers, sandpipers, and snipes of the world. University of Nebraska Press, Lincoln, Nebraska

Kålås, J. A., Byrkjedal, I. (1981). The status of breeding waders Charadrij in Norway including Svalbard. In
Runde, O. J., Barrett, R. T. (ed.) Proc. Second Nordic Congr. Ornithol., 1979. Norweg. Orn. Soc., Stavanger, p. 57-74. (In Norwegian; English summary)

Karlog, O., Elvestad, K., Clausen, B. (1983). Heavy metals (cadmium, copper, lead and mercury) in common eiders (Somateria mollissima) from Denmark. Nord. Vet.-Med 35: $448-451$

Khalid, R. A. (1980). Chemical mobility of cadmium in sediment-water systems. In: Nriagu, J. O. (ed.) Cadmium in the environment. Part I: Ecological cycling. John Wiley and Sons. New York, p. 257-304

Kirchgessner, M., Schwarz, F. J., Grassmann, E., Steinhart, H (1979). Interactions of copper with other trace elements In: Nriagu, J. O. (ed.) Copper in the environment. Part II Health effects. John Wiley and Sons, New York, p. $433-472$

Kjellström, T. (1979). Exposure and accumulation of cadmium in populations from Japan, the United States, and Sweden Environ. Health Perspect. 28: 169-197

Kjellström, T., Nordberg, G. F. (1978). A kinetic model of cadmium metabolism in the human being. Environ. Res. 16: 248-269

Kus, B. E., Ashman, P., Page, G. W., Stenzel, L. E. (1984). Agerelated mortality in a wintering population of dunlin. Auk 101: 69-73

Liebscher, K., Smith, H. (1968). Essential and nonessential trace elements. Arch. environ. Health 7: 881-890

Liljefors, M., Pettersson, J., Bengtsson, T. (1985). Recruitment areas for migrants trapped at Ottenby Bird Observatory. Spec. Rept, Ottenby Bird Observatory No. 5, Degerhamn, Sweden

Lindberg, P. (1983). Food choice, hunting success and energy expenditure of peregrines Falco peregrinus during the breeding season in Sweden, with comparative data on food choice of gyrfalcons Falco rusticolus. MS in: Lindberg, P., Relations between the diet of Fennoscandian peregrines Falco peregrinus and organochlorines and mercury in their eggs and feathers, with a comparison to the gyrfalcon Falco rusticolus. Ph. D. thesis, Dept. Zool., Univ. Gothenburg, Sweden

Maedgen, J. L., Hacker, C. S., Schroeder, G. D., Weir, F. W. (1982). Bioaccumulation of lead and cadmium in the royal tern and sandwich tern. Archs environ. Contam. Toxicol. 11: 99-102

Martin, J. H., Elliot, P. D., Anderlini, V. C., Girvin, D. Jacobs, S. A., Risebrough, R. W., Delong, R. L., Gilmartin, W G. (1976). Mercury-selenium-bromine imbalance in premature parturient California sea lions. Mar. Biol. 35: 91-104

Matsubara-Khan, J. (1974). Compartmental analysis for the evaluation of biological half-lives of cadmium and mercury in mouse organs. Environ. Res. 7: 54-67

Mead, C. J. (1985). 'Age'. In: Campbell, B., Lack, E. (ed.) A dictionary of birds. T. \& A. D. Poyser, Calton, p. 5-7

Mearns, R. (1982). Winter occupation of breeding territories and winter diet of peregrines in South Scotland. Ornis Scand. 13: 79-83

Moore, J. W., Ramamoorthy, S. (1984). Heavy metals in natural waters. Applied monitoring and impact assessment. Springer Verlag, New York

Murton, R. K., Westwood, N. J. (1977). Avian breeding cycles. Clarendon Press, Oxford

Neathery, M. W. (1981). Metabolism and toxicity of cadmium in animals. In: Nriagu, J. O. (ed.) Cadmium in the environment. Part II: Health effects. John Wiley and Sons, New York, p. 553-581

NERC (1983). Contaminants in marine top predators. Nat. Enviran. Res. Coun., Tech. Publ. Ser. C. No. 23, Swindon 
Nicholson, J. K. (1981). The comparative distribution of zinc, cadmium and mercury in selected tissues of the herring gull (Larus argentatus). Comp. Biochem. Physiol. 68 C: 91-94

Nicholson, J. K., Osborn, D. (1983). Kidney lesions in pelagic seabirds with high tissue levels of cadmium and mercury. J. Zool., Lond. 200: 99-118

Nicholson, J. K., Kendall, M. D. Osborn, D. (1983). Cadmium and mercury nephrotoxicity. Nature, Lond. 304: 633-635

Nie, N. H., Hull, C. H., Jenkins, J. G., Steinbrenner, K., Bent, D. H. (1975). SPSS: statistical package for the social sciences (2nd edn). McGraw-Hill Book Company, New York

Nørrevang, A. (1959). The migration patterns of some waders in Europe, based on the ringing results. Vidensk. Medd. Dansk Naturhist. Foren. 121: 181-222

Nriagu, J. O. (1979). Global inventory of natural and anthropogenic emissions of trace metals to the atmosphere. Nature, Lond. 279: 409-411

Nyholm, E. (1985). Metaller i däggdjur och fảglar. Litteraturstudie. Nat. Swed. Environ. Prot. Bd., PM 1986. Solna, Sweden

Ohlendorf, H. M., Anderson, D. W., Boellstorff, D. E., Mulhern, B. M. (1985). Tissue distribution of trace elements and DDE in brown pelicans. Bull. environ. Contam. Toxicol. 35: 183-192

Page, G., Whitacre, D. F. (1975). Raptor predation on wintering shorebirds. Condor 77: 73-83

Parslow, J. L. F. (1973). Mercury in waders from the Wash. Environ. Pollut. 5: 295-304

Pettersson, J., Sandström, A., Johansson, K. (1986). Wintering areas of migrants trapped at Ottenby Bird Observatory. Spec. Rept. Ottenby Bird Observatory No. 6, Degerhamn, Sweden

Portenko, L. A. (1959). Studien an einigen seltenen Limicolen aus dem nördlichen und östlichen Sibirien. II. Der Sichelstrandläufer Erolia ferruginea (Pontopp.). J. Orn., Lpz. 100: $141-172$

Prater, A. J. (1980). Migration patterns of waders (Charadrii) in Europe, In: Nöhring, R. (ed.) Acta XVII Congr. Internat. Ornithol. Verlag der Deutschen OrnithologenGesellschaft, Berlin, p. 507-511

Prater, A. J., Marchant, J. H., Vuorinen, J. (1977). Guide to the identification and ageing of Holarctic waders. British Trust for Ornithology, Beech Grove, Tring

Raspor, B. (1980). Distribution and speciation of cadmium in natural waters. In: Nriagu, J. O. (ed.) Cadmium in the environment. Part I: Ecological cycling. John Wiley and Sons, New York, p. 147-236

Ronald, K., Frank, R. J., Dougan, J., Frank, R., Braun, H. E. (1984). Pollutants in harp seals (Phoca groenlandica). II. Heavy metals and selenium. Sci. Total Environ. 38: $153-166$

Ryan, P. G., Cooper, J. (1985). Waders (Charadrii) and other coastal birds of the northwestern Cape Province, South Africa. Bontebok 4: 1-8

Sabbioni, E., Marafante, E., Amantini, L., Ubertalli, L., Pietra, R. (1978). Cadmium toxicity studies under long term-low level exposure (LLE) conditions. I. Metabolic patterns in rats exposed to present environmental dietary levels of $\mathrm{Cd}$ for two years. Sci. Total Environ. 10: 135-161

Salomons, W., Förstner, U. (1984). Metals in the hydrocycle. Springer-Verlag, Berlin

Scanlon, P. F. (1982). Wet and dry weight relationships of mallard (Anas platyrhynchos) tissues. Bull. environ. Contam. Toxicol. 29: 615-617

Simpson, W. R. (1981). A critical review of cadmium in the marine environment. Prog. Oceanogr. 10: 1-70
Siri, W. E. (1949), Isotopic tracers and nuclear radiations with applications to biology and medicine. McGraw-Hill Book Company, New York

SOF (1978). Sveriges fåglar Swed. Orn. Soc., Stockholm

Soikkeli, M. (1967). Breeding cycle and population dynamics in the dunlin (Calidris alpina). Annales Zool. Fennici 4: 158-198

Soikkeli, M. (1970). Mortality and reproductive rates in a Finnish population of dunlin Calidris alpina. Ornis Fennica 47: 149-158

Sokal, R. R., Rohlf, F. J. (1981). Biometry (2nd edn). W H. Freeman \& Co., San Francisco

Staav, R. (1983). Longevity list of birds ringed in Sweden. Fauna och flora 78: 265-276. (In Swedish; English summary)

Stanley, P. J., Minton, C. T. D. (1972). The unprecedented westward migration of curlew Sandpipers in autumn 1969. Brit. Birds 65: 365-380

Sulkava, S. (1968). A study on the food of the Peregrine, Falco p. peregrinus Tunstall, in Finland. Aquilo, Ser, Zool. 6: $18-31$

Summers, R. W., Cooper, J., Pringle, J. S. (1977). Distribution and numbers of coastal waders (Charadrii) in the southwestern Cape, South Africa, summer 1975-1976. Ostrich 48: 85-97

Svärdson, G. (1953). Visible migration within Fenno-Scandia. Ibis 95: 181-211

Svartengren, M., Elinder, C.-G., Friberg, L., Lind, B. (1986). Distribution and concentration of cadmium in human kidney. Environ. Res. 39: 1-7

Symonds, F. L., Langslow, D. R. (1984). Geographical origins and movements of shorebirds using the Firth of Forth. Ring. Migr. 5: 145-152

Talbot, V., Simpson, C. (1983). The validity of using arithmetic means to summarize environmental pollution data. Chem. Aust. 50: 156-158

Task Group on Metal Accumulation (1973). Accumulation of toxic metals with special reference to their absorption, excretion and biological half-times. Environ. Physiol. Biochem. 3: 65-107

Tsuchiya, K., Sugita, M. (1971). A mathematical model for deriving the biological half-life of a chemical. Nord. Hyg. Tidskr. 52: 105-110

Underwood, E. J. (1977). Trace elements in human and animal nutrition (4th edn). Academic Press, New York

Uspenski, S. M. (1969). Die Strandläufer Eurasiens. Die Neue Brehm-Bücherei 420, A. Ziemsen Verlag, Wittenberg

Voous, K. H. (1973). List of Recent Holarctic bird species. Non-passerines. Ibis 115: 612-638

Ward, P. (1979). Heavy metals in waders. Inst. Terrest. Ecol, Nat. Environ. Res. Coun., Ann. Rept. 1978, Cambridge, p. $56-57$

White, C. M., Cade, T. J. (1971). Cliff-nesting raptors and ravens along the Colville River in Arctic Alaska. Living Bird 10: 107-150

White, D. H., Finley, M. T (1978). Uptake and retention of dietary cadmium in mallard ducks. Environ. Res. 17: $53-59$

White, D. H., Finley, M. T., Ferrell, J. F. (1978). Histopathologic effects of dietary cadmium on kidneys and testes of mallard ducks. J. Toxicol. Environ. Health 4: $551-558$

White, D. H., King, K. A., Prouty, R. M. (1980). Significance of organochlorine and heavy metal residues in wintering shorebirds at Corpus Christi, Texas, 1976-1977 Pestic. Monit. J. 14: 58-63

Whitelaw, D. A., Underhill, L. G., Cooper, J., Clinning, C. F. 
(1978). Waders (Charadrii) and other birds on the Namib Coast: counts and conservation priorities. Madoqua 11: $137-150$

Whitfield, D. P. (1985). Raptor predation on wintering waders in southeast Scotland. Ibis 127: 544-558

Widdowson, E. M., Dickerson, J. W. T. (1964). Chemical composition of the body. In: Comar, C. L., Bronner, F. (ed.) Mineral metabolism, Vol. 2 A. Academic Press, New York, p. $1-247$

Wiemeyer, S. N., Lamont, T G., Locke, L. N. (1980). Residues of environmental pollutants and necropsy data for Eastern
United States ospreys, 1964-1973. Estuaries 3: 155-167

Wilson, J. R., Czajkowski, M. A., Pienkowski, M. W. (1980). The migration through Europe and wintering in West Africa of curlew sandpiper. Wildfowl 31: 107-122

Wolff, W. J. (1983). Estuarine benthos. In: Ketchum, B. H. (ed.) Estuaries and enclosed seas. Ecosystems of the world 26, Elsevier Scientific Publishing Co., Amsterdam, p. 151-182 Woodwell, G. M., Rich, P. H., Hall, C. A. S. (1973). Carbon in estuaries. In: Woodwell, G. M., Pecan, E. V. (ed.) Carbon and the biosphere. Proc. Brookhaven Symp. Biol. 24, Natl. Tech. Inform. Serv., Springfield, Virginia, p. 221-240

This article was submitted to the editor; it was accepted for printing on October 28,1986 